\title{
Biomarkers in heart failure: ready for use?
}

\author{
Bogdan Solnica \\ Department of Clinical Biochemistry, Jagiellonian University Medical College, Kraków, Poland
}

\section{Correspondence to:}

Bogdan Solnica, MD, PhD, Katedra Biochemii Klinicznej, Uniwersytet Jagielloński, Collegium Medicum, ul. Kopernika 15a, 31-501 Kraków, Poland, phone: +48124214006 fax: +48 124210673 , e-mail: mbsolnic@cyf-kr.edu.pl Received: June 21, 2015 Accepted: June 22, 2015 Conflict of interest: none declared. Pol Arch Med Wewn. 2015; 125 (6): 398-399

Copyright by Medycyna Praktyczna, Kraków 2015
Heart failure (HF) is currently one of the most challenging health care problems worldwide and will remain so in the nearest future. Despite years of successful research and advances in clinical practice, the diagnosis of $\mathrm{HF}$, determination of prognosis, and selection of treatment still need to be improved. The currently available diagnostic tools including laboratory tests and echocardiography or other imaging studies do not fully meet clinicians' needs. The rapidly growing area of HF research are biomarker studies. Biomarkers may be defined as substances that can be measured in body fluids and that are involved in HF, which is considered to be a multiorgan disease process rather than affecting only the heart or circulation. Thus biomarkers may reflect abnormal regulation of organ systems and tissue damage associated with HF. ${ }^{1,2}$ Currently, more than 100 established and candidate biomarkers are being investigated to evaluate their usefulness in the diagnosis, prognosis, and treatment of HF. The majority of papers published over the last 20 years report on the prognostic value of one or more evaluated biomarkers. ${ }^{2}$ The most extensively investigated biomarkers in HF include natriuretic peptides (B-type natriuretic peptide [BNP], N-terminal proBNP, mid-regional pro-atrial natriuretic peptide), soluble ST2 (sST2), growth differentiation factor 15, cardiac troponins, markers of inflammation and oxidative stress ( $C$-reactive protein [CRP], tumor necrosis factor $\alpha$, interleukin 6 , myeloperoxidase [MPO]), markers of extracellular matrix modelling (matrix metalloproteinases [MMP-2, MMP-3, MMP-9] and their tissue inhibitors (TIMPs, galectin 3, collagen precursors), neurohormones (endothelin 1, urocortin 1, arginine vasopressin represented by copeptin, adrenomedullin (ADM) represented by midregional pro-ADM), renal tubular markers (neutrophil gelatinase-associated lipocalin [NGAL], N-acetyl-beta-d-glucosaminidase, kidney injury molecule 1), and, recently, microRNAs. ${ }^{1-3}$

In the current issue of the Polish Archives of Internal Medicine, Rywik et al. ${ }^{4}$ reported the prognostic value of novel biomarkers in comparison with broad biochemical evaluation in HF patients.
The study evaluated routine laboratory tests (complete blood count, electrolytes, liver function tests, creatinine with estimated glomerular filtration rate and uric acid) and a set of selected biomarkers (growth hormone, insulin-like growth factor 1, NT-proBNP, sST2, MMP-9, CRP, procollagen III, MPO, NGAL). Prognostic value of the evaluated tests was assessed based on the 6 months follow-up of 179 patients with HF applying either all-cause death or heart transplantation as primary endpoint and secondary composite endpoint including death, heart transplantation, justified antiarrhythmic cardioverter-defibrilator intervention or hospitalization due to HF. Most of biomarkers selected by the authors for evaluation were the subject of numerous studies assessing their diagnostic and prognostic value (TABLE). ${ }^{1-3,5}$ The results obtained in the study by Rywik et al. support the final conclusion that biomarkers do not outweigh routine laboratory evaluation in the prediction of outcome in HF patients. They found that only uric acid and sodium levels were independent predictors of the primary endpoint and eGFR of secondary endpoint. Of the evaluated biomarkers only NT-proBNP and sST2 were associated with prognosis in the studied group.

The results and conclusions presented by Rywik et al., ${ }^{4}$ being in contrast to many published data, ${ }^{1,2}$ are interesting and not surprising. Some explanations may be found in the study itself. The follow-up period was short and the number of studied patients achieving particular endpoints was small. An important issue is the proper selection of cut-off values for the diagnostic/prognostic biomarker evaluation. In this study, it could be difficult because many of the obtained test results were within or near the reference ranges. Some of the biomarkers selected for evaluation were extensively studied and found to have high prognostic value whereas others were not. Nonetheless, almost all biomarkers evaluated by Rywik et al. ${ }^{4}$ are considered emerging or candidate markers. Only natriuretic peptides and cardiac troponins are recommended in clinical practice guidelines developed by European and American cardiology societies for the use in $\mathrm{HF}^{6,7}$ It should be stressed 
TABLE Reported diagnostic and prognostic value of heart failure biomarkers ${ }^{1,2}$

\begin{tabular}{lll} 
Biomarker & Diagnosis & Prognosis \\
BNP / NT-proBNP & ++++ & ++++ \\
\hline cardiac troponins & + & ++++ \\
\hline sST2 & + & ++++ \\
\hline GDF-15 & - & +++ \\
\hline TNF-a & - & ++ \\
\hline CRP & - & ++ \\
\hline IL-6 & - & ++ \\
\hline MP0 & - & ++ \\
\hline galectin 3 & - & +++ \\
\hline endothelin 1 & - & ++ \\
\hline copeptin & - & ++ \\
\hline MR-proADM & - & ++++ \\
\hline NGAL & - & ++++ \\
\hline RDW & - & ++++ \\
\hline microRNAs & + & - \\
\hline
\end{tabular}

Abbreviations: BNP, B-type natriuretic peptide; CRP, C-reactive protein; GDF-15, growth differentiation factor 15; IL-6, interleukin 6; MP0, myeloperoxidase; MR-proADM, midregional pro-adrenomedullin; NGAL, neutrophil gelatinase-associated lipocalin; NT-proBNP, N-terminal pro-B-type natriuretic peptide; RDW, red blood cell distribution width; sST2, soluble ST2; TNF-a, tumor necrosis factor a

that the same guidelines strongly recommend the use of routine laboratory tests in the diagnosis of $\mathrm{HF}$ and assessment of disease severity and prognosis. ${ }^{6,7}$ All the other biomarkers mentioned above and presented in the TABLE are still being investigated in numerous experimental and clinical trials, which sometimes yield conflicting results. ${ }^{1-3}$ The use of $\mathrm{HF}$ biomarkers still requires analytical improvement, mostly standardization of immunoassays and harmonization of results ${ }^{5}$ as well as a comprehensive diagnostic/prognostic evaluation..$^{1,2}$ Although much evidence has been reported, there is still much to be discovered in the field of biomarkers in HF. The huge scientific effort reflected by a rapidly growing number of publications on biomarkers will soon undoubtedly provide added value to the still imperfect diagnosis of HF. ${ }^{2,8}$ The study reported by Rywik et al. ${ }^{4}$ is part of this research and debate.

\section{REFERENCES}

1 van Kimmenade RRJ, Januzzi JL, Jr. Emerging Biomarkers in Heart Failure. Clin Chem. 2012; 58: 127-138.

2 Schmitter D, Cotter G, Voors AA. Clinical use of novel biomarkers in heart failure: towards personalized medicine. Heart Fail Rev. 2014; 19 369-381.

3 Pouleur AC. Which biomarkers do clinicians need for diagnosis and management of heart failure with reduced ejection fraction? Clin Chim Acta. 2015; 443: 9-16.

4 Rywik TM, Janas J, Klisiewicz A, et al. Prognostic value of novel biomarkers compared with detailed biochemical evaluation in patients with heart failure. Pol Arch Med Wewn. 2015; 125: 434-442.

5 Clerico A, Passino C, Franzini M, Emdin M. Cardiac biomarker testing in the clinical laboratory:Where do we stand? General overview of the methodology with special emphasis on natriuretic peptides. Clin Chim Acta. 2015; 443: 17-24.

6 The Task Force for the Diagnosis and Treatment of Acute and Chronic Heart Failure 2012 of the European Society of Cardiology. Developed in collaboration with the Heart Failure Association (HFA) of the ESC. ESC Guidelines for the diagnosis and treatment of acute and chronic heart failure 2012. Eur Heart J. 2012; 33: 1787-1847.

72013 ACCF/AHA Guideline for the Management of Heart Failure, A Re port of the American College of Cardiology Foundation/ American Heart Association Task Force on Practice Guidelines. J Am Coll Cardiol. 2013; 62 e147-e239.

8 Bayes-Genis A, Ordonez-Llanos J. Multiple biomarker strategies for risk stratification in heart failure. Clin Chim Acta. 2015; 443: 120-125. 